\title{
大尺度充气式桁架太阳能阵列设计及动力学分析
}

\author{
卫剑征 张鹏飞 马瑞强 陈雪岩 谭惠丰 \\ (哈尔滨工业大学特种环境复合材料技术国家重点实验室 哈尔滨 150080)
}

\begin{abstract}
摘要: 提出充气式妳架展开大尺度太阳能阵列结构设计方法, 具有热固化复合材料充气铰实现高效折叠收拢, 以及展开后提 高结构刚度的特性。研制了原理样机, 并进行了地面展开试验, 验证了充气展开及其充气式析架的性能。可展开析架结构设 计特色是将可折叠充气支撑管与刚性碳纤维杆相结合使用, 减少热功耗并提高结构刚度。考虑可展开析架的腹管设计以及材 料固化前后对大尺度结构动态特性的影响, 对太阳能阵列的半结构进行有限元建模, 并采用 Block Lanczos 法求解结构的模 态, 计算分析了不同激励位置时结构阻尼对结构的振动响应。结果表明, 所设计的充气式栴架展开大尺度太阳能阵列采用热 固化方法, 能实现大尺度结构从柔性大转动展开向高刚度结构特性的改变; 且对低频结构具有振动抑制效果, 其振动响应能 受到激励幅值和载荷的影响, 并显著改变结构的动力学性能。
\end{abstract}

关键词: 可展开析架; 充气; 太阳能阵列; 大尺度; 柔性

中图分类号: V19

\section{Design and Dynamics of Large-scale Solar Arrays with Inflatable Mast}

\section{WEI Jianzheng ZHANG Pengfei MA Ruiqiang CHEN Xueyan TAN Huifeng}

(National Key Laboratory of Science and Technology for Advanced Composites in Special Environments,

Harbin Institute of Technology, Harbin 150080)

\begin{abstract}
A structural design method of large-scale solar array deployed was presented by an inflatable mast, which has the characteristics of thermal curable composite inflatable hinges to achieve efficient folding, and to improve structural stiffness after deployment. The principle prototype is developed, and the ground deployment test is carried out to verify the performance of deployment of the inflatable mast. The structural design of deployable mast is characterized by the combination of foldable inflatable tubes and carbon fiber beams, which can reduce thermal power consumption and improve structural stiffness. Considering the web design of deployable mast and the influence of rigidizable material on the dynamic characteristics of large-scale structures, the finite element model of the semi-structure of solar array is established, and the modal of the structure is solved by Block Lanczos method. The vibration response of structure under different excitation positions is calculated and analyzed. The results show that the large-scale solar array deployed by inflatable mast can change the characteristics of large-scale structure from flexible large rotation deployment to high stiffness structure by thermal curing method, and the structure has the effect of vibration suppression. These structure responses are affected by excitation amplitude and load, and the dynamic performance of the structure is significantly changed.
\end{abstract}

Key words: deployable mast; inflatable; solar arrays; large-scale; flexible

\section{0 前言}

空间可展开结构是 20 世纪 60 年代后期随着航 天科技的发展而诞生的一种新型宇航机构/结构。该 结构一般需要经历三个阶段和三状态; 即发射阶段 的折叠状态、展开阶段的体系转换不稳定状态、工 作阶段的稳定展开状态 ${ }^{[1]}$ 。这需要研究可展开机构/

20190306 收到初稿, 20190809 收到修改稿
结构设计、材料适应性、结构动力学及其稳定性分 析等, 所以该结构/机构的研究具有力学、材料、机 械与宇航等学科交叉特点, 目前也是宇航结构工程 领域的研究难点之一。美国最早进行了可展开结构 研究与应用, 欧洲太空局和日本等在空间可展开结 构领域的研究也取得重要成果 ${ }^{[2-3]}$ 。而我国对空间可 展开结构的研究起步于 20 世纪 90 年代, 开展了结 构概念、设计分析、模型研制、理论研究等方面工 作，但大型空间可展开结构体系还没投入应用 ${ }^{[1]}$ 。 然而, 航天器正朝着大尺度、轻量化和模块化的方 
向发展。大尺度可展开的太阳能阵列是未来航天器 重要应用领域之一。它不仅可以为航天器在轨运行 提供动力, 同时也可构建空间大型太阳能电站。同 样, 太阳能阵列结构的空间展开设计以及展开后结 构动力学特性对航天器的姿态及控制有重要的影 响。张神贝等 ${ }^{[4]}$ 进行了微重力环境下可展开复合材 料桁架的模态测试, 分析了斜加劲索的预应力对结 构刚度的影响。李天等 ${ }^{[5]}$ 用有限元方法研究了腹管 的布局设计对三角形桁架的扭转变形和屈曲的影 响。熊波等 ${ }^{[6]}$ 进一步采用多尺度分析方法讨论了复 合材料接头的可靠性对桁架的影响。而对于展开铰 的研究, 游斌弟等 ${ }^{[7]}$ 研究了铰链对太阳能阵列展开 过程接触碰撞的扰动影响。美国喷气推进实验室在 1999 年地面测试了 12 块两侧对称的合成孔径雷达 (Synthetic aperture radar, SAR)的基板展开可靠性(每

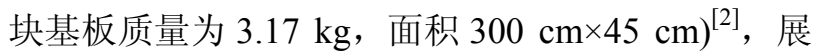
开铰链为对扣的柱壳薄钢片, 修正了仿真分析模型。 之后, 美国国防高级研究计划局(Defense advanced research projects agency, DARPA)对 $300 \mathrm{~m}$ 长新型天 基雷达进行了可行论证 ${ }^{[8-10]}$, 并采用充气展开热固 化技术完成了 $12 \mathrm{~m}$ 长的工程样机地面验证, 后续研 究暂未见公开报道。王新栋等 ${ }^{[11]}$ 采用辛算法模拟了太 阳能接收器的二维简化模型的展开过程, 研究了求解 过程中约束违约问题。卫剑征等 ${ }^{[12-13]}$ 采用分段多腔控 制体积法仿真分析了平行充气支撑管展开太阳帆板 的动力学过程。这些设计与分析主要是展开二维平面 结构, 关于三维空间桁架展开平面基板研究较少。

鉴于热固化柔性复合材料具有优异的结构特性 和灵活的设计性 ${ }^{[14]}$, 于是, 本文提出了充气展开热 固化方法构建大尺度充气式栴架式太阳能阵列的三 维空间结构, 给出大尺度太阳能阵列的结构设计, 然后分析了该大尺度充气式桁架式太阳能阵列模 态, 最后进行展开后结构受到扰动激励的结构响应。 由于这种大尺度、大面积的空间展开结构设计复杂, 本文主要是从结构设计的可行性方面进行分析, 为 未来大型空间可展开系统应用提供参考。

\section{1 太阳能阵列结构设计}

\section{1 结构设计}

大尺度充气式桁架太阳能阵列设计有 $32 \times 2$ 块 基板组成, 每块基板的面积为 $400 \mathrm{~cm} \times 235 \mathrm{~cm}$, 厚 度为 $23 \mathrm{~mm}$, 面密度是 $680 \mathrm{~g} / \mathrm{m}^{2}$ 。为了展开并能长 期在轨支撑太阳能阵列, 本文把基板对称分为两组, 采用无关节的充气铰展开方法和复合材料热固化技 术。本文参考文献[15]在轨成功展开方法, 采用充
气驱动展开 $\mathrm{V}$ 形折叠充气铰(图 1), 采用两条平行 展开方向、分段间隔的粘扣控制基板上下往复展开 运动的速度和次序。依据文献[16]中热塑性的二级 相变刚化技术，本文设计两个玻璃化转变温度 $\left(T_{\mathrm{g}}\right)$, 即常温型与潜伏型。第一个 $T_{\mathrm{g}}$ 是适用于地面折叠展 开测试的温度 $60{ }^{\circ} \mathrm{C}$, 第二个 $T_{\mathrm{g}}$ 在轨工作设计为 $130{ }^{\circ} \mathrm{C}$ 。首先, 在地面进行加热到第一个 $T_{\mathrm{g}}$ 温度附 近, 树脂软化进行折叠收拢; 在轨后将折叠结构进 行加热, 并辅助充气压力到使之回复到展开状态, 然后提高温度至第二个 $T_{\mathrm{g}}$ 引发潜伏型固化剂与未 固化树脂产生二阶段固化, 提高材料强度和刚度。这 里, 为了进一步提高大尺度太阳能阵列的抗弯刚度, 本文设计了热固化的三角形充气式桁架，它是由纵向 的充气支撑主管、两侧支撑侧边管和充气铰构成。其 中一侧桁架支撑侧边管是由热固化的斜向充气腹管 和垂直与支撑主管的热固化横向充气管组成，另一侧 的桁架支撑侧边管是由碳纤维斜向腹管和垂直与主 管的碳纤维横管组成。当充气铰展开基板后，基板背 面的充气式桁架中斜向热固化充气腹管和横向充气 管，以及热固化支撑主管进行加温后充气膨胀，并驱 动展开桁架另一侧可转动的碳纤维管(收拢时有一半 体积嵌入到基板背面里)，构成等腰三角形的桁架结 构。图 1 是整个太阳能阵列的结构的相邻两单元, 整 个结构展开到位后长度为 $150 \mathrm{~m}$, 如图 $2 \mathrm{~h}$ 所示。

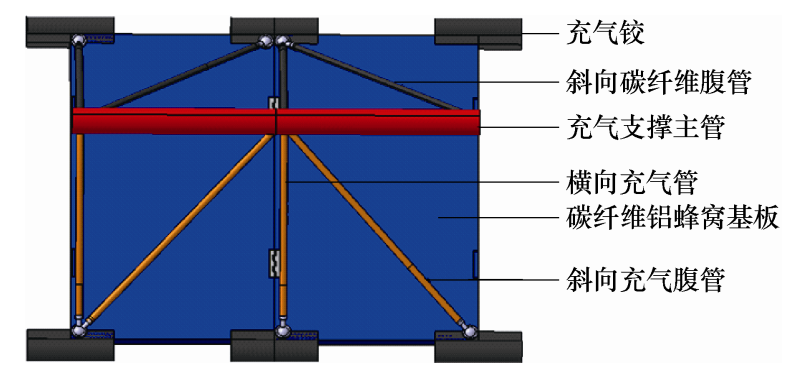

图 1 太阳能阵列的相邻基板结构组成

\section{2 结构展开方案及其地面验证}

大尺度充气桁架式太阳能阵列的展开是整个结 构设计的核心，如果这个柔性折叠桁架不能展开基 板, 或不能展开为三维空间桁架, 那么在轨任务是 不能实现的。本文将阵列以卫星本体为中心设计为 两组，两侧对称向外同步展开，每组基板的短边与 两个充气铰, 长边上由两铰链连接。首先对基板两 侧热固化充气铰进行加热, 加热到第一个 $T_{\mathrm{g}}$, 然后 启动减压阀通过 $7.2 \mathrm{MPa}$ 气瓶分别同时向充气铰内 充气，该段充气铰内压力增强，体积膨胀产生的力 矩，促使第一折开始剥离管壁粘扣，逐渐有序展开 (图 $2 \mathrm{a} \sim 2 \mathrm{~d}$ )。第一折完全展开后, 超过临界压力, 第二个充气铰开始充气、压力增加体积膨胀，依次 展开后续基板(图 2e、2f), 然后两侧基板都展开到 


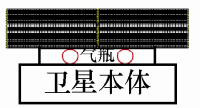

(a) 初始状态

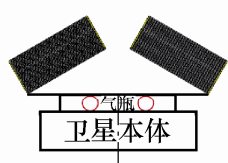

(b) 第一折充气展开

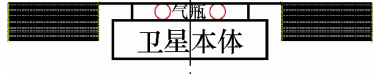

(d) 第一折展开到位

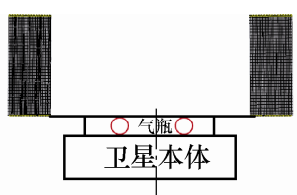

(c) 第一折展开 $90^{\circ}$ (e) 第二折展开 $90^{\circ}$

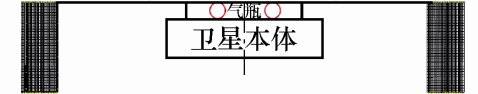

(

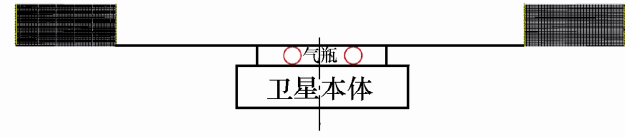

(f) 第二折展开到位

(g) 基板完全展开

(h) 背面的三角形桁架充气展开成型

图 2 太阳能阵列展开过明程组图

位(图 2g), 最后用另一高压气瓶对桁架充气，充气 式支撑主管, 以及充气式斜腹管和横管带动碳纤维 杆逐步展开, 形成三维稳定的空间结构(图 $2 \mathrm{~h}$ )。当 展开完毕后, 采用电加热与光照结合策略加热至第 二个 $T_{\mathrm{g}}$ 温度, 实现结构的长久支撑。

因为图 2 展开设计方案是变质量的运动展开, 即收拢部分被充气展开减少一块基板, 所以收拢部 分展开到位的瞬间将对整个卫星本体产生一个扰动 的冲击力矩。由于冲击力矩与时间和展开角速度有 关, 本文假设每个展开单元(每块基板 $(8.1 \mathrm{~kg})$ 和每个 展开桁架单元(约 $1.4 \mathrm{~kg}$ )) 是均质的, 即质量 $m=9.5$ $\mathrm{kg}$, 宽度 $l=2.35 \mathrm{~m}$ 。据试验观察, 假设展开到位瞬 时冲击作用时间 $\Delta t=0.8 \mathrm{~s}$, 卫星本体简化为一个质 点, 依据质点系的动量矩定理, 计算三种不同展开 角速度时的冲击力矩 $M_{d}$

$$
M_{d}=\frac{(32-n) n m w l^{2}}{\Delta t}
$$

式中, $n$ 是展开到位基板的数量, $w$ 为基板的平均 展开角速度。

图 3 为基板展开过程中冲击力矩变化, 结果表 三种情况下均为第 16 块基板展开时整个结构产生 最大冲击力矩。当展开角速度为 $0.02 \mathrm{rad} / \mathrm{s}$ 时, 最大

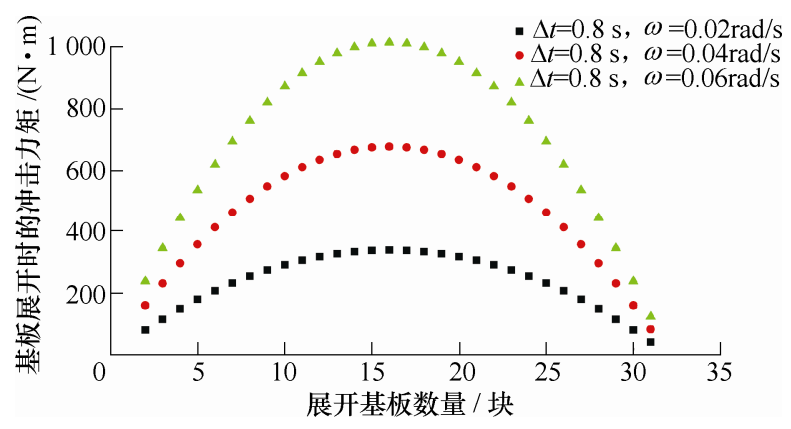

图 3 太阳能阵列的基板展开过程中冲击力矩变化
冲击力矩为 $338.5604 \mathrm{~N} \cdot \mathrm{m}$; 角速度为 $0.04 \mathrm{rad} / \mathrm{s}$ 时最 大冲击力矩为 $677.1208 \mathrm{~N} \cdot \mathrm{m}$, 角速度为 $0.06 \mathrm{rad} / \mathrm{s}$ 时最大冲击力矩为 $1015.681 \mathrm{~N} \cdot \mathrm{m}$ 。

为了说明充气展开方案可行性, 试制了 10 块厚 度 $10 \mathrm{~mm}$ 薄板, 每块板的面积 $58 \mathrm{~cm} \times 30 \mathrm{~cm}$, 基板 的上下两侧通过直径 $60 \mathrm{~mm}$ 的充气管(替代材料), 该管外壁有两个平行间断分布的粘扣进行展开力和 次序的控制。基板的背面设计有充气式桁架，10 块 板完全展开后, 对充气式桁架进行充气, 形成三维 结构, 提高结构的面外弯曲刚度和扭转刚度。试验 展开组图如图 4 所示。

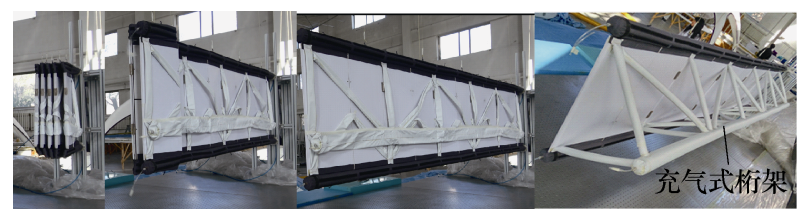

图 4 原理样机地面展开试验

\section{2 结构模态分析}

\section{1 结构建模及计算方法}

对于空间大尺度太阳能阵列展开后的结构模态 特性对于评估结构设计很有意义。展开后太阳能阵 列模型包括基板、充气式桁架中支撑主管、斜向充 气腹管和横向充气管、碳纤维腹管与横管、以及充 气铰构成。其中基板近似为薄板模型, 单元类型为 缩减积分的 S4R 壳单元; 桁架中的充气式支撑主管、 腹管和横管, 以及碳纤维管采用梁单元模型处理; 充气铰采用耦合接触方式等效处理, 采用 B31 单元, 建立太阳能阵列结构的基板以及充气式桁架的有限 元模型如图 5 所示。由于结构关于卫星本体对称分 布, 于是建立结构的 $1 / 2$ 模型, 分析整体结构的模 
态特征。单侧展开状态的太阳能阵列结构模型长度 为 $75 \mathrm{~m}$, 展开阵列由 32 块周期单元而成, 且每块 基板背面设计一组支撑结构, 相邻基板间隙为 10 $\mathrm{mm}$, 由两个铰链展开到位后锁定。本文主要对比分 析可展开桁架的充气展开部分处于充气状态(固化 前)和固化后两个状态下结构的动态特性, 以及基板 背面的析架结构有无腹管对结构动态特性的影响。

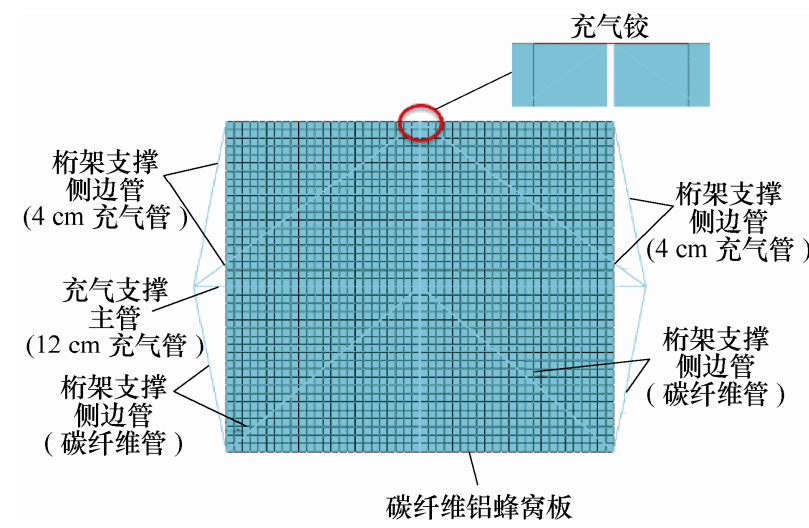

图 5 太阳能阵列展开后的两个单元的有限元模型

太阳能阵列结构的基板材料用碳纤维铝蜂窝 板, 厚度为 $23 \mathrm{~mm}$ 。充气结构部分采用正交的单 层芳纶纤维与热塑性树脂组合而成, 当结构展开 后通过内部导电薄膜对树脂进行加热固化。充气 式桁架中的充气支撑主管直径设计为 $12 \mathrm{~cm}$ 、厚度
为 $25 \mu \mathrm{m}$; 充气展开铰采用直径 $10 \mathrm{~cm}$ 、厚度为 $25 \mu \mathrm{m}$ 的芳纶纤维复合材料; 桁架中充气的斜向 腹管与横向管设计为直径 $4 \mathrm{~cm}$ 、厚为 $25 \mu \mathrm{m}$ 。作 为碳纤维的桁架支撑侧边的腹管和横管直径 1.5 $\mathrm{cm}$ 、厚度为 $2 \mathrm{~mm}$ 。等效材料参数见表 1 , 结构单 元如表 2 所示。

表 1 材料参数表

\begin{tabular}{ccccc}
\hline 结构部件 & 材料 & $\begin{array}{c}\text { 线密度 } \\
/(\mathrm{kg} / \mathrm{m})\end{array}$ & 泊松比 & 弹性模量 $/ \mathrm{MPa}$ \\
\hline 直径 $4 \mathrm{~cm}$ & 固化前 & 0.072 & 0.32 & 75.0 \\
斜向充气管 & 固化后 & 0.452 & 0.32 & 1500 \\
直径 $10 \mathrm{~cm}$ & 固化前 & 0.180 & 0.32 & 75.0 \\
横向充气管 & 固化后 & 1.113 & 0.32 & 1500 \\
直径 $12 \mathrm{~cm}$ & 固化前 & 0.216 & 0.32 & 75.0 \\
充气支撑主 & 固化后 & 1.356 & 0.32 & 1500 \\
管 & & & & \\
栴架支撑侧 & & & & \\
边直径 1.5 & 碳纤维 & 0.147 & 0.33 & 124290 \\
cm 斜向腹 & & & & \\
管和横管 & & & & \\
& 上下碳 & $1600($ 面密 & 0.26 & 104200 \\
& 纤维板 & 度, $\left.\mathrm{kg} / \mathrm{m}^{2}\right)$ & & \\
碳纤维铝 & & & & $E_{1}=E_{2}=0.162$ \\
蜂窝基板 & 铝蜂窝 & $3.112($ 面密 & & \\
& 芯子 & 度, $\left.\mathrm{kg} / \mathrm{m}^{2}\right)$ & & $E_{3}=808.29$ \\
& & & & $G_{12}=0.061$ \\
& & & $G_{13}=G_{23}=151.93$ \\
\hline
\end{tabular}

表 2 充气桁架式太阳能阵列展开结构动力特性分析

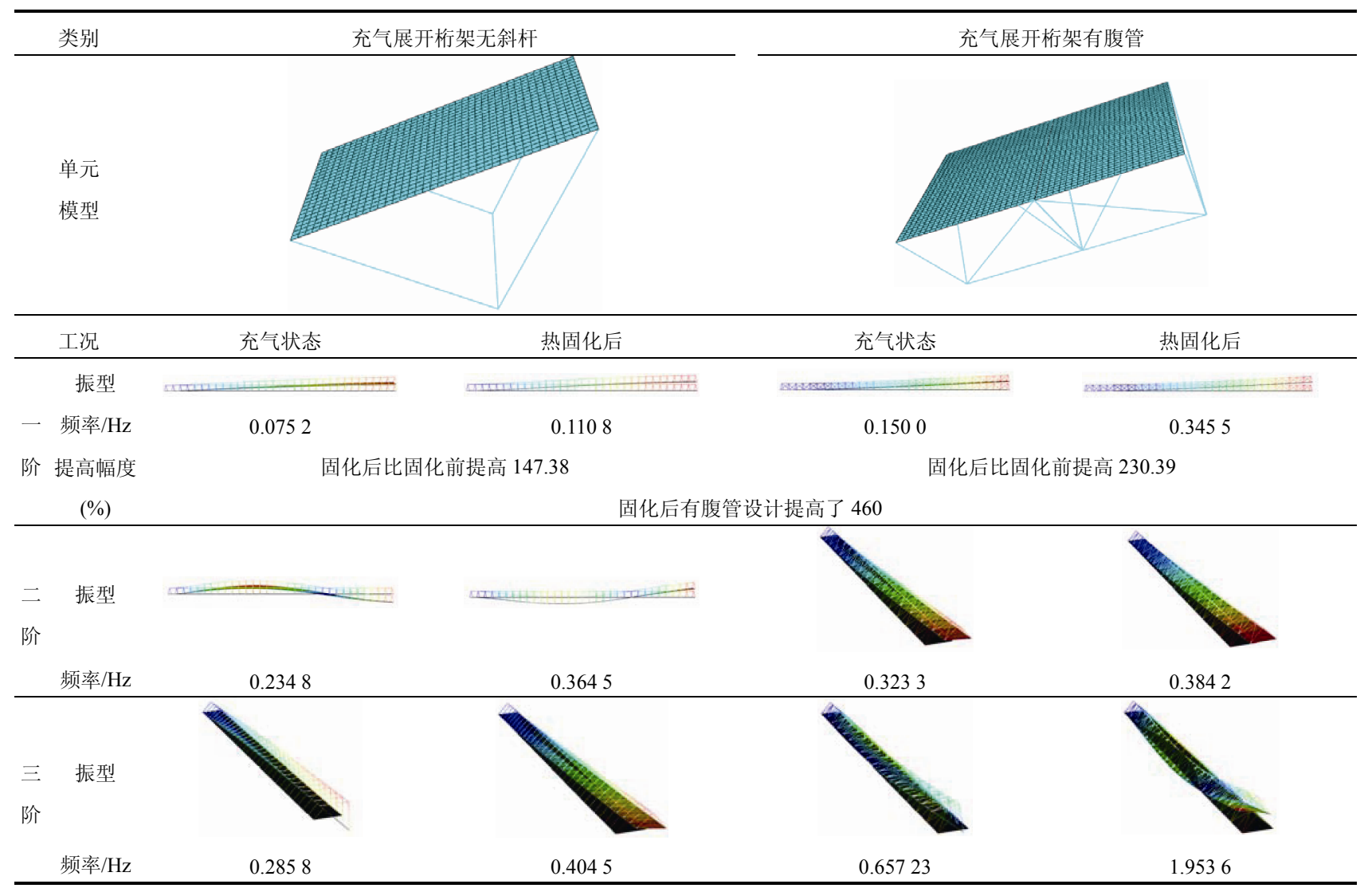


对于展开后大尺度充气式桁架结构的模态分 析, 主要计算整个结构的固有频率及相应的模态阵 型等参数。考虑充气管结构的热固化材料有阻尼特 性, 采用 d'Alembert 原理直接考虑作用于质量上全 部力的平衡, 沿位移自由度方向作用的力有内充气 体压力以及运动引起的惯性力、阻尼力和弹性力。 即结构的动力学平衡方程为

$$
M \ddot{u}(t)+C \dot{u}(t)+K u(t)=P(t)
$$

式中, $\boldsymbol{M}$ 为结构质量矩阵, $\boldsymbol{C}$ 为结构阻尼矩阵, $\boldsymbol{u}(t)$ 、 $\dot{\boldsymbol{u}}(t)$ 和 $\ddot{\boldsymbol{u}}(t)$ 分别为结构节点的位移矢量、速度矢量 和加速度矢量, $\boldsymbol{P}(t)$ 为节点的内充气压载荷矢量。 采用 Block Lanczos 法对于整个结构的模态及振型 进行分析。

\section{2 结构模态分析}

采用该模型对展开后的太阳能阵列结构进行模 态特性分析, 对比充气式析架有无增强斜向腹管、 充气结构中树脂是否固化对其特性的影响, 分析结 果见表 2 。结果表明, 没有增强斜向腹管、未固化 时太阳能阵列结构的基频为 $0.0752 \mathrm{~Hz}$, 第一阶模 态振型为与单元基板垂直的方向弯曲振动; 第 2 阶 频率为 $0.2348 \mathrm{~Hz}$, 模态振型同样为与单元基板垂 直的方向的 2 阶弯曲振动; 第三阶固有频率为 $0.2858 \mathrm{~Hz}$, 阵型为绕基板展开方向的扭转振动。当 充气式桁架结构的无增强斜向腹管热固化后, 由于 热固性树脂模量的显著提高, 弹性模量增加到 1.5 $\mathrm{GPa}$ 。从表 2 中可得出固化后结构的基频为 0.1108 $\mathrm{Hz}$, 是未固化状态的 1.4738 倍, 其模态振型与未固 化结构的情况相同, 即第一阶、第二阶阵型为沿与 单元基板垂直的方向弯曲振动, 第三阶阵型为绕基 板展开方向的扭转。最后, 还可得到含增强斜向腹 管的太阳能阵列结构的振动特性, 其第一阶和第三 阶模态振型与不含斜杆结构的相同, 但是其第二阶 模态振型不再相同, 而是变为沿基板所在平面的一 阶弯曲振动; 通过增加增强斜杆可使得固化后结构 的基频增加了 2.119 倍, 其基频为 $0.3455 \mathrm{~Hz}$, 相比 无增强斜杆、且无热固化结构, 其基频增加了 4.6 倍。

图 6 为充气式太阳能阵列结构在热固化过程中 结构固有频率的变化规律, 从图 6 中可以看出, 对 于热固化前的结构, 通过增加斜向腹管, 结构基频 增加一倍; 对于固化后的结构, 通过设计斜向腹管, 结构基频增加 2.3 倍。另外, 随着固化中材料弹性 模量的增加, 结构的基频呈现对数函数增加趋势。 因此，采用热固化方式以及对桁架结构增加设计斜 向腹管, 可有效的增大太阳能阵列结构的基频, 使 大尺度结构充气展开后的结构刚度更高。

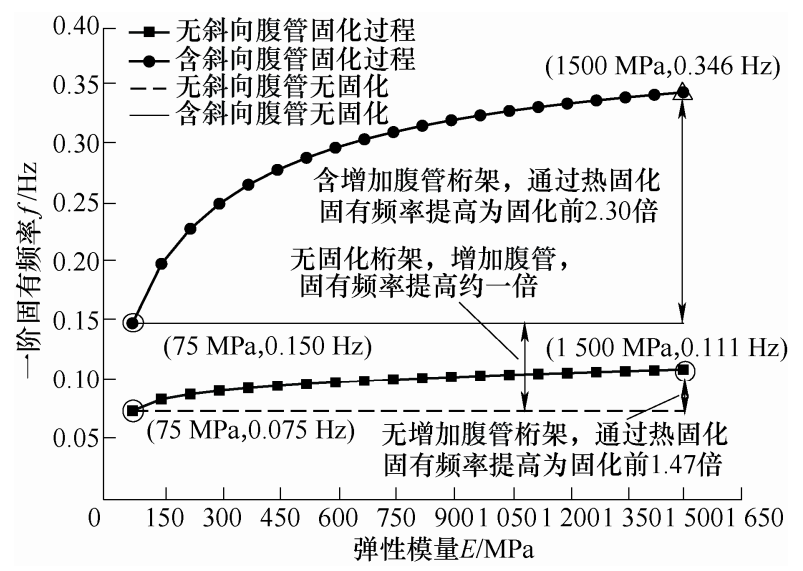

图 6 太阳能阵列刚化过程中结构固有频率的变化

\section{3 结构响应分析}

因为大尺度的空间热固化结构需要在轨调姿利 用太阳光进行辅助热固化, 所以, 本文进一步讨论 了卫星姿态因外部激励出现振动响应。同时对比结 构有无阻尼时对大尺度结构抑制振动效果和程度。 考虑到热固化充气管的纤维复合材料特性, 预先设 计粘弹性阻尼材料铺层是有效的方法，本文设计用 阻尼比 $1 \%$ 的粘弹性材料作为阻尼层, 分析阻尼层 对于充气展开桁架结构振动特性的影响。太阳能阵 列的半结构在激励作用下的动态响应通过模态振型 叠加法进行分析, 整个结构的变形可由各阶模态振 型组合计算得到，即

$$
\{u\}=\sum_{i=1}^{\infty} \partial_{i}\left\{\phi_{i}\right\}
$$

式中, $\partial_{i}$ 为结构各阶振型 $\left\{\phi_{i}\right\}$ 的标量因子。求解动 态响应采用线性摄动分析方法。

依据文献[17]中桁架的激励方式，激励幅值选 为 $1.2 \mathrm{~N}$, 模拟瞬时产生的激励作用时间为 $0.21 \mathrm{~s}$ 。 本文对太阳能阵列结构受中部激励 1 和自由端处激 励 2(图 7)两种载荷下的强迫振动为典型工况, 仿真 分析预设粘弹性阻尼材料层对有无固化、有无增强 腹管的振动抑制程度。根据上节中得到的太阳能列 阵结构的模态振型, 可以得到前三阶模态振型中测 点 1 和观测点 2 均出现了较大的位移, 于是作为结 构振动响应的观测点。对比分析有无阻尼情况下结 构中点及自由端端点的动态响应的幅度。

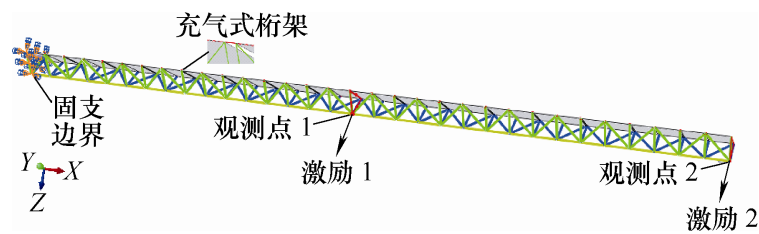

图 7 太阳能阵列结构激励位置 
采用上述方法仿真分析了含增强斜向腹管固化 前后、无增强斜向腹管固化前后的四种结构状态分 别受到激励 1 和激励 2 作用下的强迫振动, 观测预 设有无复合材料阻尼层情况下充气式桁架结构的动 态响应, 图 8 11 分别得到四种结构状态观测点的 时域函数。结果表明, 结构预设阻尼层后, 整个结 构的最大幅度出现明显降低, 表 3 为不同情况下的 结构响应最大幅度对比。
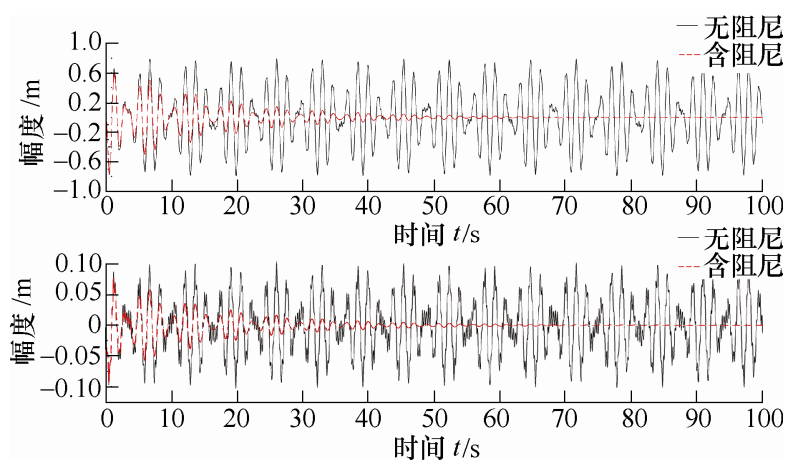

图 8 固化后有腹管的第一(上)、二阶(下)弯曲模态响应
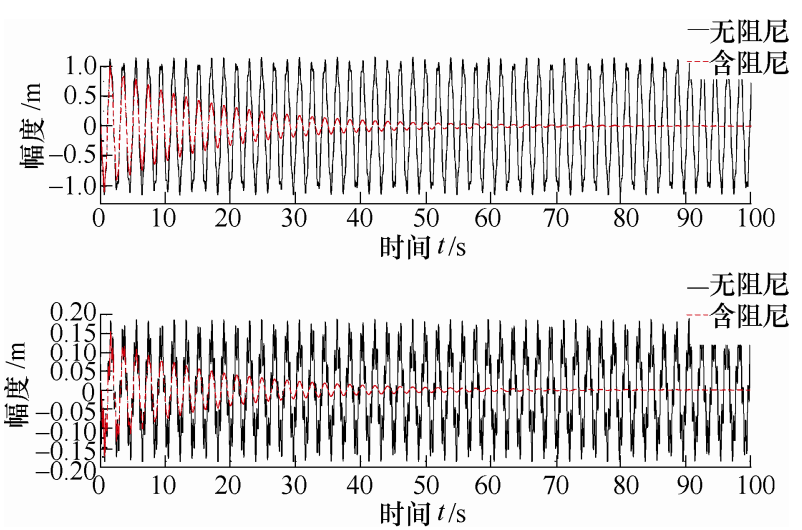

图 9 固化后无腹管的第一 (上)、二阶(下)弯曲模态响应
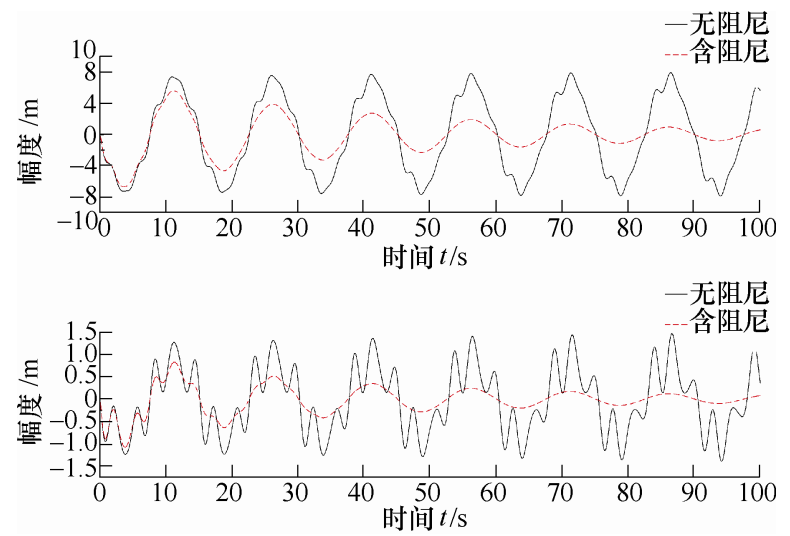

图 10 固化前有腹管的第一(上)、二阶(下)弯曲模态响应

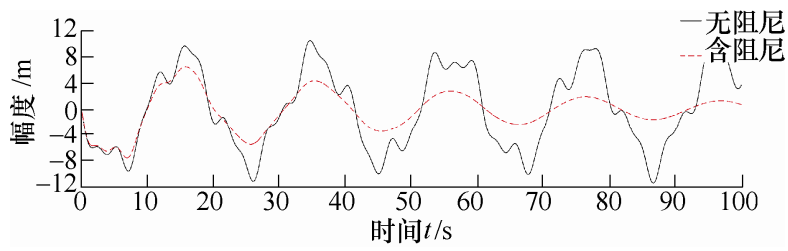

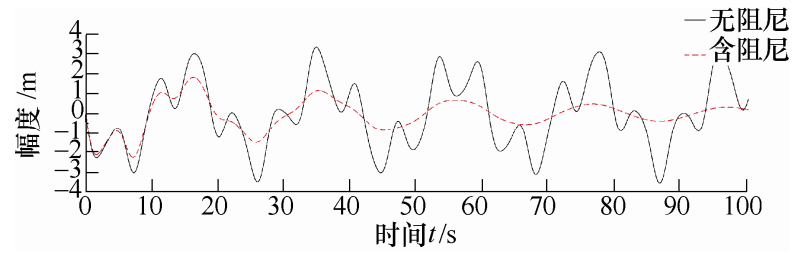

图 11 固化前无腹管的第一(上)、二阶(下)弯曲模态响应

表 3 固化前后的结构响应对比

\begin{tabular}{|c|c|c|c|c|c|}
\hline \multirow{2}{*}{\multicolumn{2}{|c|}{ 结构类型 }} & \multicolumn{2}{|c|}{ 一阶结构响应/m } & \multicolumn{2}{|c|}{ 二阶结构响应 $/ \mathrm{m}$} \\
\hline & & 无阻尼 & 有阻尼 & 无阻尼 & 有阻尼 \\
\hline \multirow{2}{*}{ 固化后 } & 有腹管 & 0.796 & 0.611 & 0.104 & 0.076 \\
\hline & 无腹管 & 1.155 & 1.102 & 0.188 & 0.155 \\
\hline \multirow{2}{*}{ 未固化 } & 有腹管 & 7.933 & 5.621 & 1.465 & 0.828 \\
\hline & 无腹管 & 10.531 & 6.524 & 3.347 & 1.818 \\
\hline
\end{tabular}

对比分析表明，在预设有阻尼层的情况下，充 气桁架结构响应将减小, 说明阻尼层起到抑制振动 的作用, 充气结构通过热固化方式可有效的减小激 励对支撑结构的影响, 降低结构的振动响应; 其次, 充气式桁架结构通过设计斜向腹管, 也可有效的降 低激励对结构的影响, 减小结构的振动响应。综合 说明, 通过设计阻尼层的方式能有效的抑制结构响 应，使得含斜向腹管的桁架结构能在约 $20 \mathrm{~s}$ 内，实 现最大振幅减小 $50 \%$ 以上; 使得不含斜向腹管结构 在 $60 \mathrm{~s}$ 内, 结构振幅降低一半左右。此外, 还可以 得到通过设计斜向腹管, 使得结构的最大振幅从 $1.155 \mathrm{~m}$ 减小到 $0.796 \mathrm{~m}$, 降低了近 1.45 倍; 而通过 对结构进行固化, 使得结构的最大振幅从 $7.933 \mathrm{~m}$ 减小到 $0.796 \mathrm{~m}$, 降低了近 10 倍。这些结果表明, 通过增加斜向腹管、阻尼和结构固化, 可以有效地 抑制结构响应。

\section{4 结论}

(1) 提出采用热固化的充气铰驱动展开大尺 度的刚性基板, 采用斜向碳纤维腹管与充气热固 化腹管展开等腰三角形桁架, 实现大尺度多折基 板的展开方法。并通过地面原理样机验证该方法 的可行性。

(2) 针对充气式桁架展开大尺度太阳能阵列, 采用热固化方法实现结构的刚度转换, 分析了热固 化过程中结构固有频率的变化规律; 固化前结构通 过增加斜向腹管, 结构基频增加了一倍, 且随着固 化中材料弹性模量的提高, 结构的基频呈现对数函 数增加趋势。

（3）针对结构的低频特点, 讨论了预设阻尼层 对大尺度充气结构响应的影响程度, 表明阻尼层设 计能有效的抑制结构响应, 使得含斜向腹管的桁架 
结构能在约 $20 \mathrm{~s}$ 内, 实现最大振幅减小 $50 \%$ 以上, 这使大尺度结构设计具有更大的参考价值。

\section{参 考 文 献}

[1] 陈务军, 张淑杰. 空间可展开结构体系与分析导论[M]. 北京: 中国宇航出版社, 2006.

CHEN Wujun, ZHANG Shujie. Deployable space structures and analysis theory[M]. Beijing : China Astronautic Publishing House, 2006.

[2] LOPEZ B C, LOU M C, GAMA E. Deployment simulations of a fold-up synthetic aperture radar array[C]// 1999 American Institute of Aeronautics and Astronautics,

AIAA-99-1445.

[3] TAKAMATSU K A. ONODA J. New deployable truss concepts for large antenna structures or solar concentrators[J]. Journal of Spacecraft, 1991, 28: 330-338.

[4] 张祎贝, 高冀峰, 陈务军, 等. 织物复合材料空间可展 桁架模态试验与分析 [J]. 振动与冲击, 2018, 37(17): 155-160.

ZHANG Yibei, GAO Jifeng, CHEN Wujun, et al. Modal tests and analysis of a weave composite space deployable truss[J]. Journal of Vibration and Shock，2018，37(17): 155-160.

[5] 李天, 昆进涛, 樊嘉. 等腰三角形空间析架自由扭转理 论分析和试验研究 [J]. 工程力学, 2018, 35(5): 223-238. LI Tian, CHAO Jintao, FAN Jia. Theoretical and experimental study on free torsion of isosceles triangular space truss[J]. Engineering Mechanics, 2018, 35(5): 223-238.

[6] 熊波, 罗锡林, 谭惠丰. 考虑接头作用的全复合材料析 架结构多尺度分析 [J]. 工程力学, 2015, 32(8): 229-235. XIONG Bo, LUO Xilin, TAN Huifeng. Multi-scale analysis of all-composite truss considering joint effects[J]. Engineering Mechanics, 2015, 32(8): 229-235.

[7] 游斌弟, 王兴贵, 陈军. 卫星太阳阵展开锁紧过程冲击 振动[J]. 机械工程学报, 2012，38(21): 67-76.

YOU Bindi, WANG Xinggui, CHEN Jun. Vibration and impact for deployable solar array of satellite with locking hinges[J]. Journal of Mechanical Engineering, 2012, 48(21): $67-76$.

[8] GUERCI J, JASKA E. ISAT-Innovative space-based-radar antenna technology[C]// 6th IEEE Phased Array Systems and Technology Symposium, Array 2003, October 14-17, 2003, Boston, MA, Institute of Electrical and Electronics Engineers Inc. 2003: 45-51.

[9] MURPHEY T, CLIFF E, LANE S. Matching space antenna deformation electronic compensation strategies to support structure architectures[J]. IEEE Transactions on Aerospace and Electronic Systems, 2010， 46(3): 1422-1436.

[10] LANE S A, MURPHEY T W. Overview of the innovative space-based radar antenna technology program[J]. Journal of Spacecraft and Rockets, 2011，48(1): 135-166.

[11] 王新栋, 胡伟鹏, 邓子辰. 空间太阳能电站太阳能接收 器二维展开过程的保结构分析 [J]. 动力学与控制学报, 2015, 13(6): 406-409.

WANG Xindong, HU Weipeng, DENG Zichen. Structure preserving analysis of $2 \mathrm{D}$ deploying process for solar power receiver of solar power satellite[J]. Journal of Dynamics and Control, 2015, 13(6): 406-409.

[12] 卫剑征, 苗常青, 杜星文. 充气太阳帆板展开动力学数 值模拟预报 [J]. 宇航学报, 2007, 28(2): 322-327.

WEI Jianzheng, MIAO Changqing, DU Xingwen. Simulation prediction of deployment dynamics of inflatable solar array[J]. Journal of Astronautics, 2007, 28(2): 322-327.

[13] 卫剑征, 谭惠丰, 苗常青, 等. 空间折叠薄膜管的充气 展开动力学实验研究 [J]. 力学学报, 2011, 43(1): 202-207.

WEI Jianzheng, TAN Huifeng, MIAO Changqing, et al. Experimental study of inflatable deployment dynamics of folded membrane booms[J]. Chinese Journal of Theoretical and Applied Mechanics, 2011, 43(1): 202-207.

[14] 刘宇艳, 孟秋影, 谭惠丰, 等. 空间充气展开结构用刚 化材料和刚化技术的研究现状[J]. 材料工程, 2008(2): 76-80.

LIU Yuyan, MENG Qiuying, TAN Huifeng, et al. The situation of the rigidizable materials and rigidization technology used in space inflatable structures[J]. Journal of Materials Engineering, 2008(2): 76-80.

[15] WEI Jianzheng, TAN Huifeng, WANG Weizhi, et al. Deployable dynamic analysis and on-orbit experiment for inflatable gravity-gradient boom[J]. Advances in Space Research, 2015, 55: 639-646.

[16] Sun He, LIU Yuyan, TAN Huifeng, et al. A new method to improve the stability, tensile strength, and heat resistant properties of shape-memory epoxy resins: Two-stages curing[J]. Journal of Applied Polymer Science, 2014, 131(4): 1001-1007.

[17] ZHAO Haojiang, LIU Rongqiang, GUO Hongwei, et al. Model analysis and active vibration control for space deployable truss type mast[C]// 2011 IEEE International Conference on Mechatronics and Automation, August 7-10, Beijing, China: Beijing, IEEE Computer Society, 2011: 371-375.

作者简介: 卫剑征, 男, 1978 年出生, 博士, 副教授。主要研究方向为 柔性展开结构动力学、柔性壳力学。

E-mail: weijz@hit.edu.cn

谭惠丰(通信作者), 男, 1969 年出生, 博士, 教授, 博士研究生导师。 主要研究方向为柔性复合材料与结构理论与应用。

E-mail: tanhf@hit.edu.cn 